\title{
ENVIRONMENTAL RISK ASSESSMENT METHOD IN THE DEVELOPMENT OF THE ARCTIC SHELF RESOURCES
}

\author{
Solovieva N. V., Lobkovsky L. I. \\ P. P. Shirshov Institute of Oceanology, Russian Academy of Sciences (Moscow, Russian Federation)
}

\section{The article was received on December 27, 2018}

\begin{abstract}
The paper proposes a method of mathematical modeling and assessment of environmental risk for the shelf ecosystems of the Arctic seas under the combined impact of natural, anthropogenic and climatic factors. The method is based on the use of observational data, dynamic models of the ecosystem state and probabilistic ecoscreening risk assessments. The input data for the risk assessment model can be the data of field observations of the organisms' biomass or the simulating results of the ecosystem state using dynamic models. For the Arctic shelf, the data used are derived from generalized field observations of the annual variations in phytoplankton biomass within highly productive and low productive ecosystems.

The proposed approach makes it possible to move from a design-fixed (laid in the project for the development of shelf resources) value of allowable impact on the ecosystem up to its variations during the year. Such changes will be determined by variations in environmental risk. Variations of the tolerance impact on the ecosystem during the year will reduce the economic costs of maintaining environmental safety. The paper substantiates the primacy of determining the allowable risk magnitude of the ecological systems, and then the allowable intensity of anthropogenic impact, which opens up the possibility to answer the questions about principle admissibility of a certain scale accidents.

The relationship between the allowable risk for the ecosystem and the allowable probability of anthropogenic impact objectifies the value of risk assessments under emergency and the normal operating conditions of engineering systems. Using the proposed method for specific ecosystems will help to harmonize environmental and economic requirements in ensuring the environmental safety at the development of shelf resources.
\end{abstract}

Keywords: Arctic ecosystems, mathematical modeling, eco-screening models, environmental risk assessment.

This research was performed in the framework of the state assignment, theme № 0149-2019-0005.

\section{References}

1. Flint M. V. Bioresursy Arkticheskikh morei Rossii: izmeneniya pod vozdeistviem klimata i faktorov antropogennoi prirody, ekosistemnye osnovy okhrany. [Bioresources of the Arctic seas of Russia: changes under the influence of climate and anthropogenic factors, ecosystem basis of protection]. Nauchno-tekhnicheskie problemy osvoeniya Arktiki. Ros. akad. nauk. Moscow, Nauka, 2015, pp. 55-71. (In Russian).

2. Kompleksnye issledovaniya bol'shikh morskikh ekosistem Rossii. [A comprehensive study of the large marine ecosystems of Russia]. Otv. red. G. G. Matishov; Murman. mor. biol. in-t Kol'skogo nauch. tsentra RAN. Apatity, Izd-vo KNTs RAN, 2011, 516 p. (In Russian).

3. Flint M. V. 54-i ekspeditsionnyi reis nauchnoissledovatel'skogo sudna "Akademik Mstislav Keldysh" v Karskoe more. [54th expedition voyage of the research vessel "Academician Mstislav Keldysh" to the Kara sea]. Okeanologiya, 2010, vol. 50, no. 5, pp. 677-682. (In Russian).

4. Sukhanova I. N., Flint M. V., Mosharov S. A., Sergeeva V. M. Struktura soobshchestv fitoplanktona i pervichnaya produktsiya $v$ Obskom estuarii i na prilezhashchem Karskom shel'fe. [Structure of phytoplankton communities and primary products in the Ob estuary and the adjacent Kara shelf]. Okeanologiya, 2010, vol. 50, no. 5, pp. 785-800. (In Russian).
5. Sukhanova I. N., Flint M. V., Pautova L. A. et al. Phytoplankton of the western Arctic in the spring and summer of 2002: Structure and seasonal changes. Deep-Sea Res. II, 2009, vol. 56, pp. 1223-1236. DOI: 10.1016/j.dsr2.2008.12.030.

6. Flint M. V., Semenova T. N., Arashkevich E. G. et al. Struktura zooplanktonnykh soobshchestv $v$ oblasti estuarnoi frontal'noi zony reki Ob'. [The structure of zooplankton communities in the estuarine frontal zone of the Ob river]. Okeanologiya, 2010, vol. 50, no. 5, pp. 809-822. (In Russian).

7. Sukhanova I. N., Flint M. V., Sazhin A. F., Sergeeva V. M., Druzhkova E. I. Phytoplankton in the northwestern Kara Sea. Oceanology, 2015, vol. 55, no. 4, pp. 547-560. DOI: $10.7868 /$ S0030157415040140.

8. Sukhanova I. N., Flint M. V., Sergeeva V. M., Nedospasov A. A., Druzhkova E. I. Structure of phytoplankton communities in the Yenisei estuary and over the adjacent Kara Sea shelf. Oceanology. 2015, vol. 55, no. 6, pp. 844-857. DOI: 10.1134/ S0001437015060193.

9. Demidov A. B., Mosharov S. A., Makkaveev P. N. Patterns of the Kara Sea primary production in autumn: Biotic and abiotic forcing of subsurface layer. J. Mar. Sys., 2014, vol. 132, pp. 130-149. DOI: 10.1016/j. jmarsys.2014.01.014. 
10. Demidov A. B., Mosharov S. A., Gagarin V. I., Romanova N. D. Prostranstvennaya izmenchivost' pervichnoi produktsii i khlorofilla “a” v prolive Dreika v vesennii sezon. [Spatial variability of primary production and chlorophyll "a" in Drake Strait in spring season]. Okeanologiya, 2011, vol. 51, no. 2, pp. 293-306. (In Russian).

11. Demidov A. B., Mosharov S. A. Vertical distribution of primary production and chlorophyll a in the Kara Sea. Oceanology, 2015, vol. 55, no. 4, pp. 521-534. DOI: 10.1134/S0001437015040025.

12. Demidov A. B., Mosharov S. A., Artem'ev V. A. Stupnikova A. N., Simakova U. V., Vazyulya S. V. Depth-integrated and depth-resolved models of Kara Sea primary production. Oceanology, 2016, vol. 56, no. 4, pp. 515526. DOI: $10.1134 /$ S0001437016040020.

13. Mosharov S. A., Demidov A. B., Simakova U. V. Peculiarities of the primary production process in the Kara Sea at the end of the vegetation season. Oceanology, 2016, vol. 56, no. 1, pp. 84-94. DOI: 10.1134/ S0001437016010100.

14. Demidov A. B., Kopelevich O. V., Mosharov S. A. et al. Modelling Kara Sea phytoplankton primary production: development and skill assessment of regional algorithms. J. Sea Res, 2017, vol. 125, pp. 1-17. DOI: 10.1016/j.seares.2017.05.004.

15. Demidov A. B., Sheberstov S. V., Gagarin V. I., Khlebopashev $P$. V. Seasonal variation of the satellite-derived phytoplankton primary production in the Kara Sea. Oceanology, 2017, vol. 56, no. 14, pp. 91-104. DOI: $10.1134 /$ S0001437017010027.

16. Il'yash L. V., Rat'kova T. N., Radchenko I. G., Zhitina L. S. Fitoplankton Belogo morya. [The phytoplankton of the White sea]. Sistema Belogo morya. Vol. 2: Vodnaya tolshcha i vzaimodeistvuyushchie s nei atmosfera, kriosfera, rechnoi stok i biosfera. Moscow, Nauch. mir, 2012, pp. 605-639. (In Russian).

17. Belyaev V. I. Modelirovanie morskikh sistem. [Modelling of marine systems]. Kiev, Nauk. dumka, 1987, 203 p. (In Russian).

18. Belyaev V. I. Konduforova N. V. Matematicheskoe modelirovanie ekologicheskikh sistem shel'fa. [Mathematical modeling of ecological systems of the shelf]. Kiev, Nauk. dumka, 19906240 p. (In Russian).

19. Solovjova N. V. Synthesis of ecosystemic and ecoscreening modelling in solving problems of ecological safety. Ecol. Modelling, 1999, vol. 124, pp. 1-10.
20. Fleishman B. S. Osnovy sistemologii. [Basics of systemology]. Moscow, Radio i svyaz', 1982, 368 p. (In Russian).

21. Fleishman B. S. Kriterii ekologicheskoi bezopasnosti v usloviyakh neopredelennosti (ekoskriningovyi podkhod). [Criteria of ecological safety in conditions of uncertainty]. Ekoskrining: Razrabotka osnov standartov ekologicheskoi bezopasnosti. Dep. v VINITI 19.12.95, no. 3401. (In Russian).

22. Solov'eva N. V. Matematicheskoe modelirovanie s ispol'zovaniem dannykh naturnykh i distantsionnykh nablyudenii dlya otsenki sostoyaniya ekologicheskoi sistemy shel'fa. [Mathematical modeling using data from field and remote observations to assess the state of the ecological system of the shelf]. Neftyanoe khoz-vo, 2005, no. 10, pp. 16-17. (In Russian).

23. Solov'eva N. V., Lobkovskii L. I. Issledovanie chuvstvitel'nosti modeli ekosistemy shel'fa k variatsiyam ee ekologicheskikh parametrov. [Study of the sensitivity of the shelf ecosystem model to variations in its environmental parameters]. Ekologiya prom. pr-va, 2008, no. 2, pp. 18-26. (In Russian).

24. Solov'eva N. V. Modelirovanie kumulyativnogo vliyaniya vneshnikh faktorov na sostoyanie ekosistemy shel'fa i otsenka dopustimoi veroyatnosti antropogennogo vozdeistviya. [Modeling the cumulative impact of external factors on the shelf ecosystem and assessment of the permissible probability of anthropogenic impact]. Ekolog. sistemy i pribory, 2018, no. 11, pp. 23-35. DOI: 10.25791/esip.11.2018.253. (In Russian). 25. Solov'eva N. V., Lobkovskii L. I. Modelirovanie ekologo-ekonomicheskogo riska pri osvoenii shel'fa. [Modeling of environmental and economic risk in offshore development]. DAN, 2015, vol. 464, no. 3, pp. 356360. (In Russian).

26. Solov'eva N. V. Issledovaniya ekologicheskoi sistemy shel'fa Severnogo Kaspiya na osnove matematicheskogo modelirovaniya. [Studies of the ecological system of the North Caspian shelf on the basis of mathematical modeling]. Ekolog. sistemy i pribory, 2016, no. 6, pp. 21-32. (In Russian).

27. Patin S. A. Neft' i ekologiya kontinental'nogo shel'fa. [Oil and the environment of the continental shelf]. $\mathrm{V}$ 2-kh t. 2-e izd. Vol. 1: Morskoi neftegazovyi kompleks: sostoyanie, perspektivy, faktory vozdeistviya. Moscow, Izd-vo VNIRO, 2017, 326 p. (In Russian).

\section{Information about the authors}

Solovieva Natalia Vladimirovna, Doctor of Physical and Mathematical Sciences, Leading Researcher, P. P. Shirshov Institute of Oceanology, Russian Academy of Sciences (36, Nahimovskiy prosp., Moscow, Russia, 117997), e-mail: soloceanic@yandex ru.

Lobkovsky Leopold Isaevich, Doctor of Physical and Mathematical Sciences, Corresponding member of the Russian Academy of Sciences, Head of Laboratory, P. P. Shirshov Institute of Oceanology, Russian Academy of Sciences (36, Nahimovskiy prosp., Moscow, Russia, 117997), e-mail: Llobkovsky@ocean.ru.

\section{Bibliographic description}

Solovieva N. V., Lobkovsky L. I. Environmental Risk Assessment Method in the development of the Arctic shelf resources. Arctic: Ecology and Economy, 2019, no. 1 (33), pp. 50-60. DOI: 10.25283/2223-4594-2019-1-50-60. (In Russian).

(c) Solovieva N. V., Lobkovsky L. I., 2019 


\title{
МЕТОД ОЦЕНКИ ЭКОЛОГИЧЕСКОГО РИСКА ПРИ ОСВОЕНИИ РЕСУРСОВ АРКТИЧЕСКОГО ШЕЛЬФА
}

\author{
Н. В. Соловьева, Л. И. Лобковский \\ ФГБУН Институт океанологии им. П. П. Ширшова РАН (Москва, Российская Федерация)
}

Статья поступила в редакцию 27 декабря 2018 г.

Предложен метод математического моделирования и оценки экологического риска для шельфовых экосистем морей Арктики при совместном действии естественных, антропогенных и климатических факторов. Метод основан на данных наблюдений, динамических моделях состояния экосистемы и вероятностных экоскрининговых оценках риска. Обосновывается первичность определения величины допустимого риска экологических систем, а затем допустимой интенсивности антропогенного воздействия, что открывает возможность получать ответы на вопросы о принципиальной допустимости аварий определенных масштабов. Использование предложенного метода для конкретных экосистем поможет согласовать эколого-экономические требования к обеспечению экологической безопасности освоения ресурсов шельфа.

Ключевые слова: арктические экосистемы, математическое моделирование, экоскрининговые модели, оценка экологического риска.

\section{Введение}

Разработка минеральных, углеводородных и биологических ресурсов арктического шельфа требует новых подходов, создания новых технологий и техники для их безопасного освоения, учитывающих особенности региона, его повышенную чувствительность и уязвимость. Риски негативного воздействия на арктические экосистемы при разведке, добыче, транспортировке нефтяных углеводородов, при радиационном и химическом загрязнении, при развитии инвазивных процессов сказываются на всех звеньях арктических экосистем от клеточного уровня, отдельных видов организмов до структурно-функциональных характеристик экологических систем. Исследования экосистем, находящихся в условиях изменения климатических факторов, выявили нетривиальные реакции на внешние воздействия [1-16]. Условия функционирования экосистем арктических акваторий, их чувствительность и уязвимость к антропогенным воздействиям коренным образом различаются в разных морях Арктики [1]. Исследования текущего состояния, структурно-функциональных особенностей и прогноз возможных реакций экосистем на хозяйственное освоение арктического шельфа, а также климатический отклик являются основой для оценки экологического риска с после-

(с) Соловьева Н. В., Лобковский Л. И., 2019 дующей оценкой допустимого воздействия. Важнейшим в экономическом и экологическом смысле при получении таких оценок является первичность определения величины допустимого риска экологических систем, а не, наоборот, допустимой интенсивности антропогенного воздействия. Это позволяет учесть специфику задач экологической безопасности, состоящую в несимметричности требований к исключению ошибок первого и второго рода. Если имеет место ошибка первого рода, т. е. безопасная ситуация принята за ситуацию, представляющую угрозу безопасности экосистемы, то излишняя перестраховка, связанная с ложной тревогой, не так опасна (хотя влечет за собой необоснованные затраты), как ошибка второго рода, когда опасная ситуация скрыта внешним благополучием.

Если учесть, что антропогенный пресс действует на фоне существенных изменений естественных факторов различных пространственно-временны́х масштабов (от региональных до климатических), становится понятной необходимость комплексного подхода к оценке экологических рисков. В его основе лежат методы математического моделирования экологического риска с выходом на управление риском [17-21]. Методика отрабатывалась на задачах, связанных с экологическим состоянием и риском разработок шельфа [22-26]. 
Факторы, определяющие состояние арктических акваторий, и учет их в эколого-экономическом решении природно-хозяйственных задач

Необходимость разработки методов математи ческого моделирования состояния [17; 18] и риска [19] экологических систем шельфа обусловлена перспективами освоения ресурсов принципиально различных акваторий, отличающихся как гидрофизическими, гидрологическими, гидрохимическими, так и гидробиологическими параметрами и условиями их функционирования. Бо́льшая часть арктического шельфа с первичной продукцией менее $50 \mathrm{mгC} / \mathrm{M}^{2}$ в день относится к ультраолиготрофным районам, но в отдельных локальных областях продукция в сотни раз выше. Если в среднем показатели крайне низкие - 8 мгС/ $\mathrm{M}^{3}$ в сутки, то диапазон вариаций значений первичной продукции на порядок выше, от 1,12 до 66,31 мгС/м³ в сутки. Изменчивость значений биомассы фитопланктона на трансарктическом разрезе протяженностью около 6 тыс. км находит-

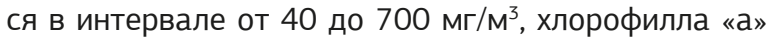

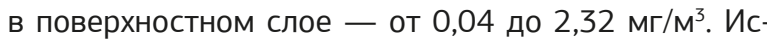
следования сотрудников Института океанологии РАН в более чем 10 экспедиционных рейсах по программе «Экосистемы российской морской Арктики» выявили различие в механизмах функционирования высокопродуктивных локальных экосистем и преобладающих сильно обедненных в продукционном отношении акваториях арктического шельфа. В частности, выявлено наличие физического барьера сильного распреснения поверхностного 5-12-метрового слоя за счет речного стока в краевые арктические моря от Карского до Чукотского. Сформированная плотностная стратификация настолько устойчива, что не преодолевается вертикальной сезонной конвекцией, а следовательно, не происходит обогащения фотического слоя биогенными элементами, участвующими в процессе фотосинтеза. Даже современные климатические изменения - увеличение безледного периода, прогрев поверхностного слоя воды - не ослабляют барьер, связанный с лимитированием фотосинтеза биогенными элементами [1]. Учет таких особенностей функционирования морских экосистем Арктики будет определять разные уровни экологического риска и, следовательно, разные уровни допустимости антропогенного воздействия, что поможет варьировать требования к уровню обеспечения надежности технологических процессов разработки минеральных ресурсов шельфа (рис. 1). Другими словами, открывается возможность оптимального согласования экологических и экономических требований, которые на практике зачастую вступают в противоречия. Предлагаемая нами методика оценки и управления риском является основой, позволяющей привести в действие экономические рычаги обеспечения экологической безопасности разработок ресурсов шельфа Арктики. Эта методика синтезирует в себе все последние до- стижения в области мониторинга экосистем, включая математическое моделирование экологических систем, экоскрининговые оценки риска и управление риском с выходом на эколого-экономические интерпретации результатов.

Оценка риска по предлагаемой методике позволяет дать ответ на вопрос о принципиальной допустимости аварий в различных акваториях арктического шельфа определенных масштабов (рис. 1). Полученные результаты могут служить основой классификации типов аварий на допустимые (с указанием допустимой вероятности осуществления) и недопустимые именно для специфики арктического региона. А это, в свою очередь, является основой определения экономического ущерба.

Существующие механизмы экономического регулирования природоохранной деятельности, включая платежи за загрязнения, штрафы и компенсации, экологическое страхование и т. д., требуют коррекции или в некоторых случаях введения новых механизмов для морского арктического региона. Совместное воздействие нескольких типов антропогенной нагрузки на высоко- и низкопродуктивные акватории Арктики может давать различный отклик и приводить к разным последствиям [2; 3; 5; 7]. Учесть главные различия арктических экосистем позволит предыдущий опыт моделирования состояния экосистем шельфа $[12 ; 14 ; 15 ; 17 ; 18 ; 22 ; 24$; 26] с учетом многомерности пространства параметров и связей между компонентами единой сложной системы, включающей антропогенную деятельность человека.

Это не значит, что в решении задач оценки риска с учетом эколого-экономических требований нужно чрезмерно детализировать изучаемые явления в ущерб целостному представлению об объекте исследования. Но и ограничивать экологическую часть общей задачи лишь тривиальными выводами о связи между загрязнениями и платежами, регламентациями и ограничениями недостаточно.

Хотя экономические требования на практике вступают в противоречия с экологическими, все же можно сформулировать подход, который позволит принимать эффективные эколого-экономические решения природно-хозяйственных задач [19; 25]. В его основе лежат методы математического моделирования экологического риска с выходом на управление риском [20; 21]. Междисциплинарность предложенного подхода позволяет использовать все достоинства смежных дисциплин и избежать недостатков каждой из них в отдельности.

\section{Математическое моделирование экологического риска для экосистем арктического шельфа}

Необходимость появления экоскринингового подхода к оценке экологического риска [20; 21] обусловлена переориентацией в области экологической безопасности с концепции абсолютной безопасно- 


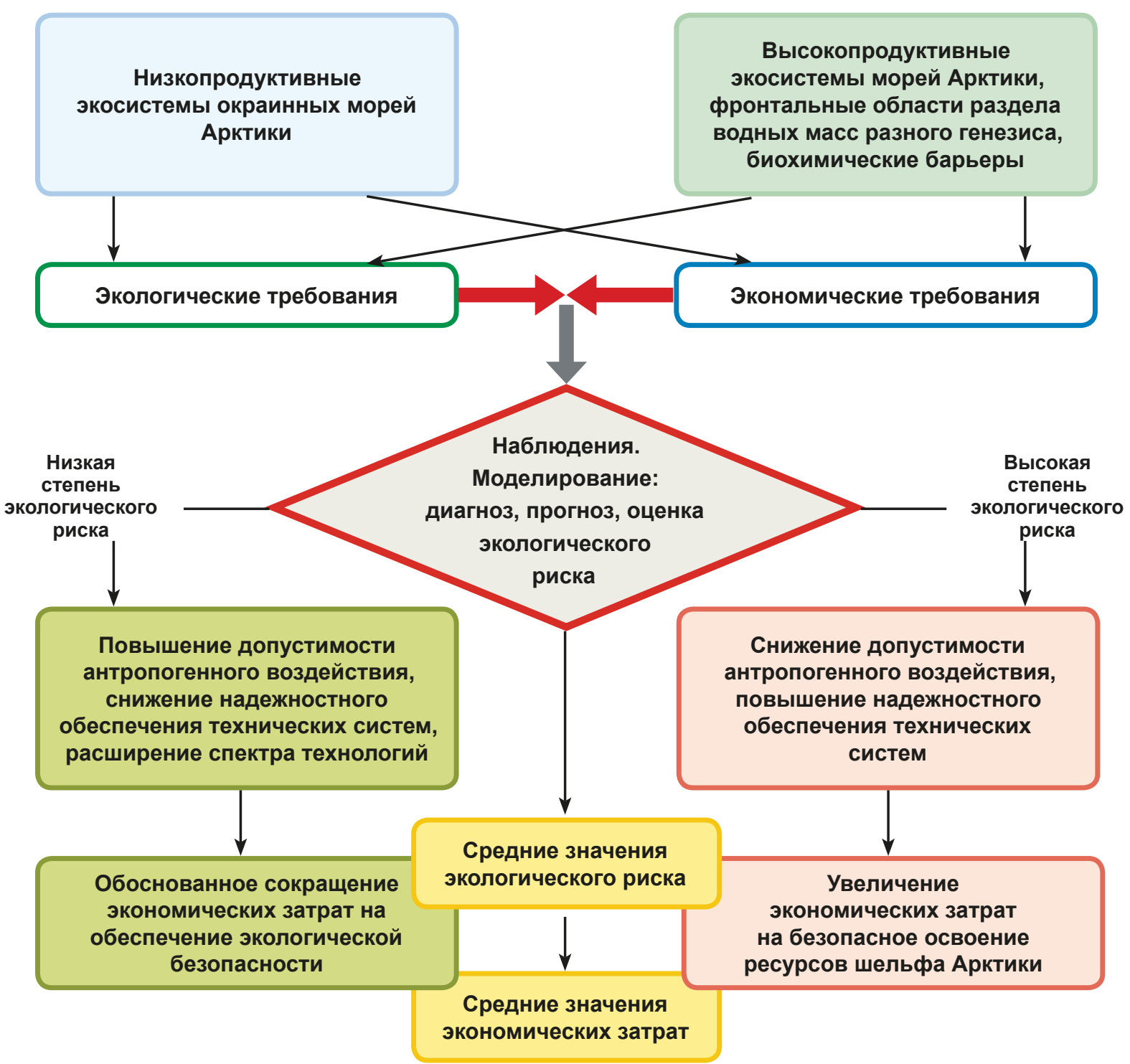

Рис. 1. Схема метода минимизации экономических затрат с сохранением экологических требований безопасного освоения ресурсов Арктики

Fig. 1. Diagram of the method for minimizing economic costs while maintaining environmental requirements in the safe development of Arctic resources

сти на концепцию приемлемого риска или на их комбинацию. Важнейшим в этом смысле является первичность определения величины допустимого риска экологической системы, а не наоборот - допустимой интенсивности антропогенного воздействия. Другими словами, нет необходимости постоянно поддерживать постоянный уровень финансирования экономических мероприятий на экологическую безопасность. Более рациональным видится подход, заключающийся в согласованном ходе вариаций риска и затрат на его предотвращение [19].

Поясним, что вряд ли стоит стремиться к созданию универсального критерия экологической безопасности для различных уровней биологической организации от экосистемы Арктики в целом до индивидуального организма. Если даже такой критерий и будет предложен, то вследствие необходимо- сти наличия в нем систем разного уровня организации он должен был бы обладать сильной степенью обобщения, так что существенные индивидуальные свойства каждого уровня организации были бы потеряны. В силу этого иерархия объектов и диапазон применимости критерия экологической безопасности должны соответствовать по крайней мере уровню организации исследуемых систем.

Вместе с тем, хотя суммарные загрязнения арктических акваторий в целом далеки от критических уровней (за исключением отдельных локальных районов), необходимо пересмотреть существующие методики оценки воздействий загрязнений на арктические экосистемы в преддверии интенсивного освоения региона. При этом нужно учесть особую чувствительность и уязвимость этих экосистем за счет меньшей степени биоразнообразия [6; 8] по 
сравнению с экосистемами умеренных и южных широт, а также принять во внимание низкую скорость разложения загрязняющих веществ в условиях пониженной температуры.

Допустимость негативного воздействия конкретного загрязнителя (токсиканта) на определенный вид организмов определяется существующей системой нормативов, недостаточность которой стала особенно очевидна в последние десятилетия. Состояние экологической системы оценивается разными группами специалистов по существующим для каждой из них нормативам: санитарно-гигиеническим, рыбохозяйственным, водопользовательским и т. д. В таких нормативах используются самые разнообразные системы индикаторов от хорошо разработанной системы предельно допустимых концентраций (ПДК), предельно допустимых выбросов и нагрузок до генетических индикаторов, используемых для определения мутагенной степени опасности. Следует отметить несовершенство каждого из подходов в отдельности. Так, нормы ПДК, защищая человека от того или иного воздействия, не рассчитаны на защиту всей экосистемы в целом. Даже если значениями ПДК пытаются нормировать какоелибо антропогенное воздействие на сумму некоторых живых организмов, то, как правило, это можно осуществить только для конкретной среды. Кроме того, такие нормы не учитывают эффекты накопления вредных веществ в организмах или последствия перехода их из одной среды в другую. Даже если оцениваются суммарные последствия нескольких одновременно планируемых проектов, выполнение норм по какому-либо одному критерию не означает экологической безопасности всей системы в целом, хотя и является необходимым ее условием. Кроме того, наличие множества частных критериев экологической безопасности затрудняет и сам выбор того или иного критерия, по которому возможно сделать заключение о степени опасности. Такая многомерность в постановке и решении задач экологии, с одной стороны, и особенности экосистем Арктики (чувствительность и уязвимость) - с другой, побуждают к разработке критерия, который с приемлемой степенью обобщения позволил бы видеть объект исследования в целом и давать оценку его экологического состояния с учетом всего разнообразия естественных условий и антропогенных воздействий.

Среди факторов, обуславливающих экологическую безопасность, можно выделить внешние и внутренние по отношению к исследуемой системе. Под внешними факторами будем понимать естественные и антропогенные, направленные на обеспечение приемлемой экологической опасности, сокращение пространства экологического риска. Внутренние факторы относятся к свойствам самой системы и связаны с устойчивостью, надежностью, резистентностью. При этом значение величины критического состояния экосистемы не является постоянным, а нелинейно зависит от внешних естественных условий, антропогенных воздействий и самого́ со- стояния экосистемы в текущий и предшествующие моменты времени.

Изложенные соображения легли в основу метода моделирования и оценки экологического риска для экосистем арктического шельфа с учетом динамики основных компонент трофической цепи в различных по естественным условиям, продуктивности и антропогенной нагрузке акваториях.

В теории экоскрининга используется понятие экологического риска как вероятности гибели особи или популяции организмов [20; 21]. Модель внутригодовой изменчивости риска основана на следующем. В реальных условиях численность особей, составляющих популяцию, закономерно меняется в течение года под воздействием естественных факторов: изменения солнечной радиации, количества питательных веществ, внутренних взаимосвязей между компонентами, лимитирующих факторов и т. д. Эти изменения могут быть получены в результате натурных измерений $[2 ; 15 ; 16]$ или численного моделирования по экосистемной модели [17; 18; 22]. Причем для оценки риска в нужную сторону (сверху [21]) необходимо учитывать самые неблагоприятные естественные условия (принцип предосторожности [27]).

В силу слабой изученности сезонной динамики биомасс основных компонент экосистем Арктики, особенно в связи с новыми трендами климатических факторов, экосистемное моделирование пока затруднено отсутствием достаточного количества натурных наблюдений. Вместе с тем качественные (с отдельными количественными значениями «реперных» величин [5; 15; 12]) выводы о динамике биомасс основных компонент трофических звеньев уже получены [16] (рис. 2). Динамика биомассы фитопланктона в более продуктивных экосистемах: Баренцево, Берингово, Чукотское (каньон Барроу) [1], Белое моря, эстуарные и склоновые фронтальные зоны [4; 6] (рис. 2а) - и низкопродуктивных: основная акватория морей Карского и Чукотского, Лаптевых, Восточно-Сибирского [3; 7; 8; 11] (рис. 26) сильно различаются [13; 15].

Отсутствие весеннего цветения фитопланктона в значительной части низкопродуктивных районов (Карское море), установленное в процессе проведения экспедиционных наблюдений [1-3], обусловлено отсутствием сезонной вертикальной конвекции (см. рис. 26). Особенностью функционирования арктических экосистем также является наличие двух сезонных максимумов биомассы фитопланктона в продуктивных экосистемах [1] (см. рис. 2а). По данным экспедиционных исследований, 23\% общей годовой первичной продукции и $32 \%$ годовой вторичной продукции формируются в склоновых и приливных фронтальных зонах арктических морей, площадь которых составляет лишь 5,5\% общей площади региона [1]. Различия в значимости высокопродуктивных локальных областей и преобладающих низкопродуктивных акваторий необходимо учитывать при планировании хозяйственного освоения ресурсов Арктики. 


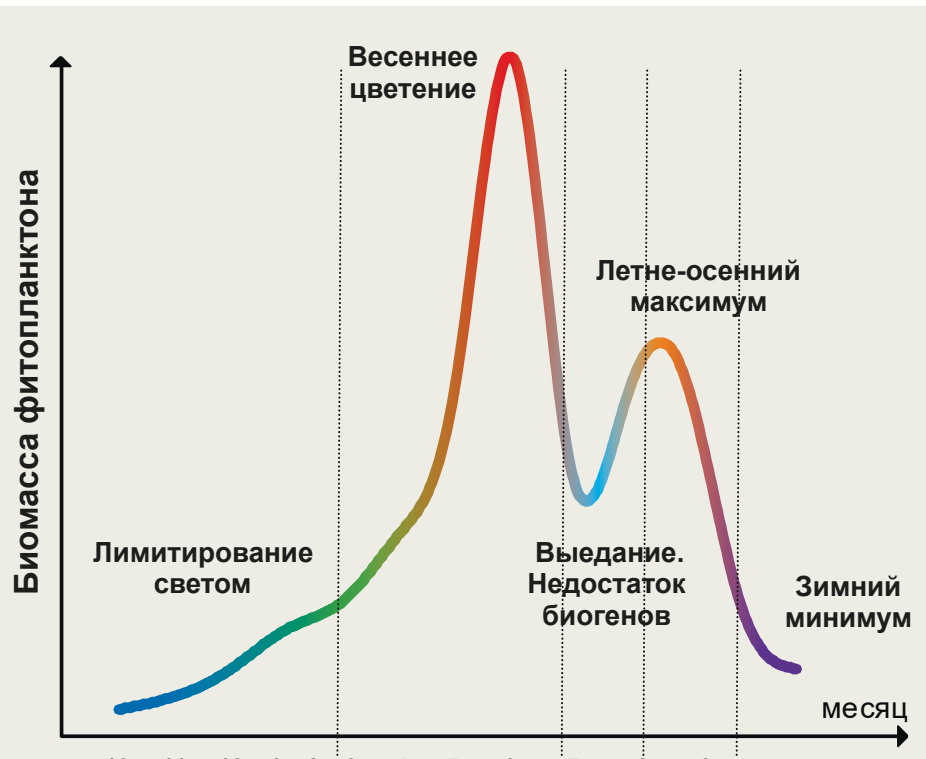

$\begin{array}{lllllllll:llll}10 & 11 & 12 & 1 & 2 & 3 & 4 & 5 & 6 & 7 & 8 & 9\end{array}$

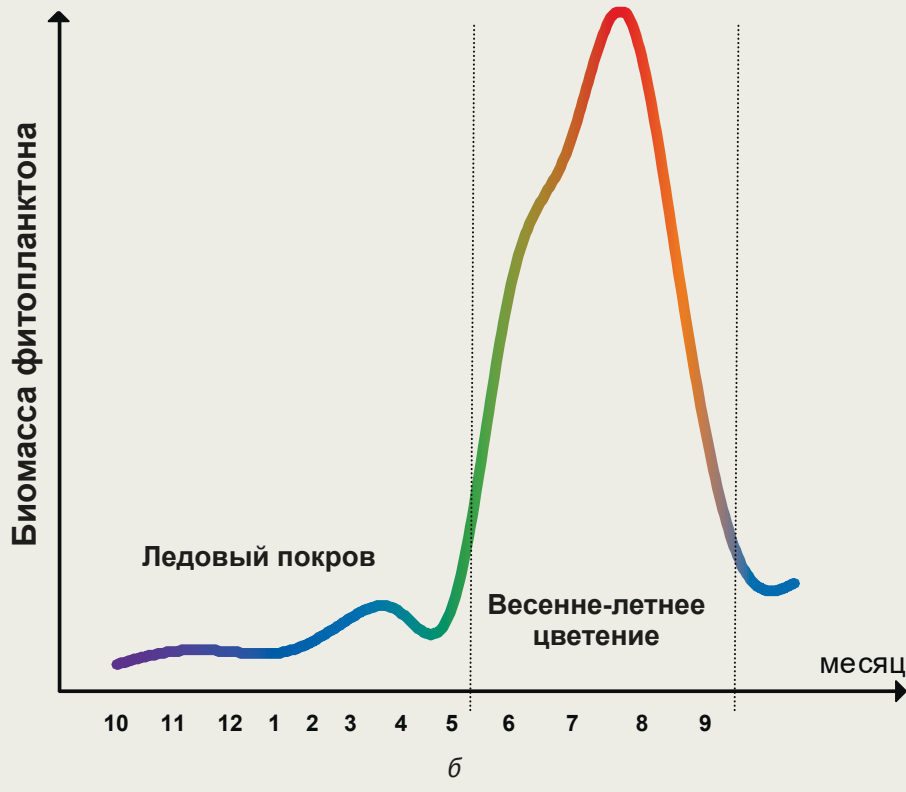

Рис. 2. Характерная сезонная динамика биомассы фитопланктона в морях Арктики по данным [16]: $a$ - Баренцево, Белое, Чукотское моря; 6 - Карское, Лаптевых, Восточно-Сибирское моря

Fig. 2. Typical season dynamics of phytoplankton biomass in the Arctic seas according to observation data [16]: $a$ - the Barents Sea, the White Sea and the Chuckchee Sea; 6 - the Kara Sea, the Laptev Sea and the East Siberian Sea

Результаты натурных наблюдений [1-16] или расчеты с помощью экосистемных моделей агрегированных компонент [17; 18; 13; 16] отражают естественные вспышки и спады биомасс (численностей) популяций. Периоды вспышек и спадов численности популяций не совпадают с календарными сезонами. Максимальные значения численности (биомассы) - для каждой $m$-й вспышки $N_{\max }$ и $m$-го спада $N_{\max }^{\prime}$. Введем значение $m=1, M$, где $M-$ количество периодов «вспышка - спад» в течение года, и значение $k=\overline{1, K}$, где $k$ - значения состояния технической системы или техпроцесса. Вероятность пребывания технической системы в состоянии $k$ обозначим $q_{k}$. Значение $M$ может меняться от года к году под воздействием естественных и антропогенных факторов, а также в зависимости от особенностей функционирования различных арктических экосистем (см. рис. 2).

В общем случае для $2 M$ внутригодовых состояний популяции «вспышка - спад» при $K$ внутригодовых состояниях технической системы (технологического режима) получена оценка внутригодового риска у в следующем виде [21; 19]:

$$
\begin{aligned}
& y \ll \sum_{k=1}^{K} q_{k} \sum_{m=1}^{M} p_{k m} y_{k m}= \\
& =\sum_{k=1}^{K} q_{k} \sum_{m=1}^{M}\left(p_{a m} y_{a m}+p_{a m}^{\prime} y_{a m}^{\prime}\right)= \\
& =\sum_{k=1}^{K} q_{k} y_{a},
\end{aligned}
$$

где $p_{a m}, y_{a m}$ и $p_{a m}^{\prime}, y_{a m}^{\prime}-$ вероятно-

сти пребывания биосистемы в $m$-м внутригодовом состоянии вспышки и спада и ее риск в них соответственно; $q_{k}$ - вероятность аварии технической системы (или в общем случае вероятность допустимой антропогенной нагрузки, предусмотренной технологическими процессами) в течение года; $y_{a}$ - вероятность экологического риска в течение года $[21 ; 19]$.

$$
\begin{aligned}
& \overline{y_{a}}=\sum_{m=1}^{M}\left(p_{a m} y_{a m}+p_{a m}^{\prime} y_{a m}^{\prime}\right), \\
& \sum_{m=1}^{M}\left(p_{a m}+p_{a m}^{\prime}\right)=1, \quad \sum_{k=1}^{K} q_{k}=1 .
\end{aligned}
$$

Значения $p_{a m}$ и $p_{a m}^{\prime}$ определяются относительной продолжительностью вспышек и спадов:

$$
p_{a m}=\frac{t_{m}}{t} ; \quad p_{a m}^{\prime}=\frac{t_{m}^{\prime}}{t} ; \sum_{m=1}^{M}\left(t_{m}+t_{m}^{\prime}\right)=t, \text { (3) }
$$

где $t_{m}, t_{m}^{\prime}$ и $t$ - продолжительности вспышек, спадов и года соответственно в произвольных единицах измерения.

Из соотношений (2) видно, что значение вероятности экологического риска в течение года - величина непостоянная, меняющаяся в диапазоне значений от 0 до 1. Это дает основание соответственно изменять предельно допустимую антропогенную нагрузку. В терминах вероятности более высокие допустимые значения 


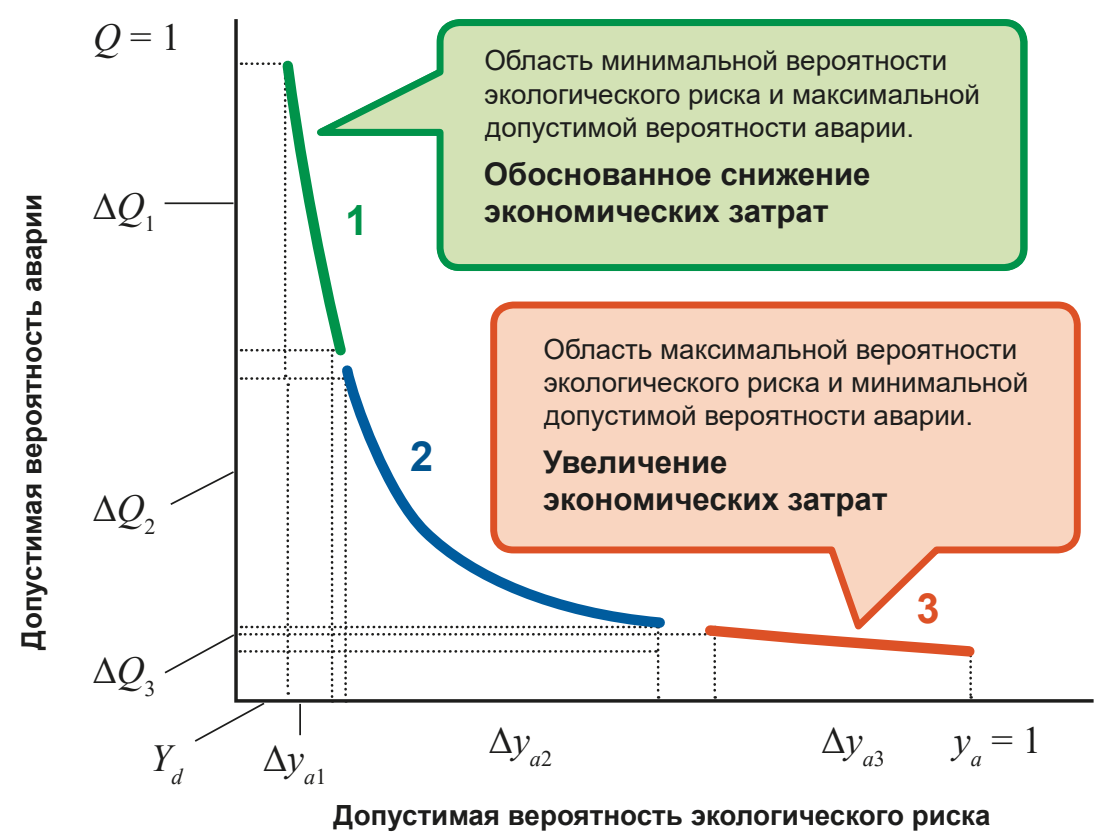

Рис. 3. Зависимость допустимой вероятности штатного и аварийного антропогенного воздействия от вероятности экологического риска

Fig. 3. The dependence of allowable probability of normal and emergency anthropogenic impact on the probability of ecological risk

вероятности антропогенного воздействия должны соответствовать меньшим значениям вероятности экологического риска, а более низкие - высоким значениям риска. Такой подход открывает эффективное согласование экологических и экономических требований. Не обязательно поддерживать постоянно экономические мероприятия для обеспечения экологической безопасности на одном и том же уровне. Можно варьировать их интенсивность в зависимости от значений экологического риска, меняющегося в течение года.

Будем использовать соотношения экоскрининга [21] для определения предельно допустимой антропогенной нагрузки на систему в следующем виде $[21 ; 25]$ :

$$
Q=\left\{\begin{array}{l}
1 \text { при } y_{a} \leq y_{d}, \\
\frac{y_{d}}{y_{a}} \text { при } y_{d}<y_{a}<1, \\
y_{d} \text { при } y_{a}=1,
\end{array}\right.
$$

где $y_{a}$ определяется оценкой (2); $Q$ - предельно допустимая вероятность антропогенных воздействий для экологической системы; $y_{d}$ - предельно допустимый экологический риск при различных видах техногенной деятельности.

Соотношения (4) позволяют перейти к управлению риском. Смысл его заключается в регулировании экономических затрат в обеспечение экологической безопасности. Качественный вид зависимости допустимой антропогенной нагрузки $q_{\partial}$ от значений экологического риска $y_{a}$ (рис. 3) показы- вает три области: две - соответствующие крайним состояниям максимального (область 3 на рис. 3) и минимального (область 1 на рис. 3) естественного экологического риска и третья - переходная область (область 2 на рис. 3).

При минимальном экологическом риске $\Delta y_{a 1}$ диапазон допустимой вероятности антропогенной нагрузки $\Delta Q_{1}$ можно увеличить до максимума, а следовательно, снизить на этом этапе экономические требования к обеспечению экологической безопасности, сократив финансирование без ущерба экосистеме. Напротив, при максимальном уровне экологического риска $\Delta y_{a 3}$ допустимая вероятность антропогенной нагрузки $\Delta Q_{3}$ должна быть сведена к минимуму, что требует увеличения экономических затрат. Гипербола средней области 2 на рис. 3 показывает диапазон изменения вероятности экологического риска $\Delta y_{a 2}$ и соответствующую ему вероятность допустимой антропогенной нагрузки $\Delta Q_{2}$, позволяя, таким образом, перераспределять экономические затраты в течение года, что повышает их эффективность и минимизирует затраты.

При известных из наблюдений (или рассчитанных по экосистемной модели) сезонных вариациях биомасс основных компонент экосистемы рассчитываются годовой ход естественного экологического риска по соотношениям (1-3) и вероятность допустимой антропогенной нагрузки $Q$ по соотношениям (4) экоскрининговой модели риска. Полученный годовой ход экологического риска $y_{a}$ позволит рассчитать соответствующий ему ход допустимой вероятности аварийных и штатных режимов техпроцессов $Q$ (рис. 4). При высоких значениях $Q$ экономические затраты в обеспечение экологической безопасности 


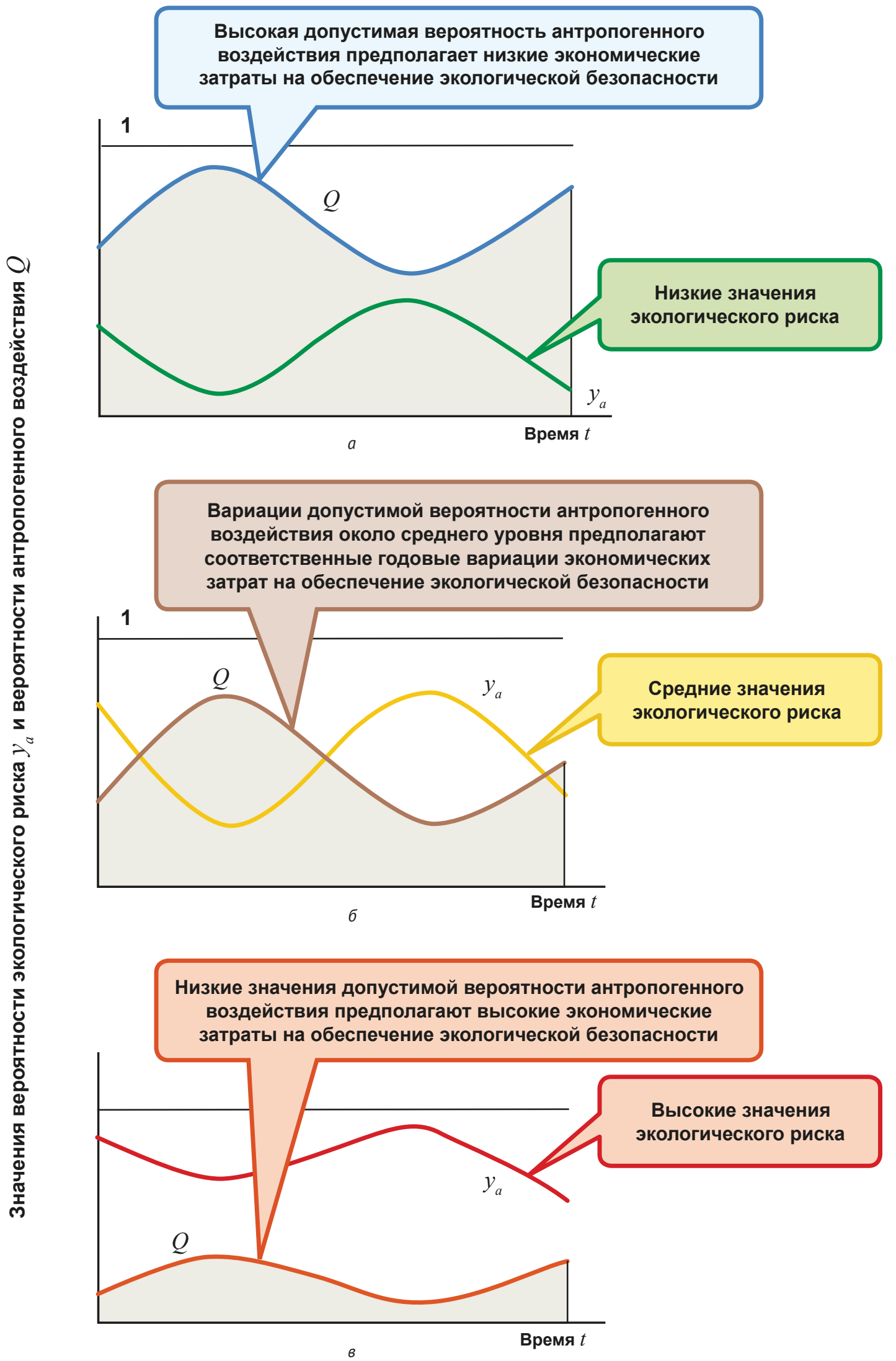

Рис. 4. Годовой ход значений допустимой вероятности антропогенного воздействия $Q$ в зависимости от допустимой вероятности экологического риска $y_{a}$, определяющий низкие (a), средние (б) и высокие (в) экономические затраты

Fig. 4. The annual value variation of allowable probability of anthropogenic impact $Q$ depending on allowable probability of ecological risk $y_{a}$ that determines the low economic costs $(a)$, the medium (G) and the high ones ( 8 ) 
могут быть минимальными (рис. 4а). Низкие значения допустимой вероятности аварии $Q$ требуют максимальных средств для предотвращения такого типа антропогенного воздействия (рис. 4в). Вариации допустимых значений $Q$ около среднего уровня (рис. 46) определяют соответствующий ход экономических мероприятий.

В свою очередь, обоснованно увеличивая или уменьшая объем финансовых вложений в экономические и технологические меры обеспечения безопасности разработок ресурсов арктического шельфа, можно также минимизировать негативное влияние последних на состояние экосистем морей Арктики и их биоразнообразие. Это может касаться не только условий осуществления того или иного антропогенного воздействия, но и значимости тех популяций, которые подвергаются риску от этого воздействия. Требования к технической системе (антропогенному воздействию) $Q_{i}$ от каждой $i$-й популяции, вообще говоря, различны. Долгоживущие популяции требуют меньших значений $Q_{i}$, чем короткоживущие. Обобщенные на случай $I$ популяций соотношения (4) позволяют определять допустимые значения вероятности аварии (или штатного режима) технической системы по отношению к $i$-й популяции. Если существование всех $I$ популяций нам одинаково дорого, то к надежности технической системы необходимо предъявлять требование допустимой годовой вероятности аварии, удовлетворяющей условию $Q=\min _{i} Q_{i}$.

\section{Заключение}

Переориентация в области экологической безопасности для морского арктического региона на концепцию приемлемого риска необходимо требует разработки методов оценки риска с учетом всех групп факторов естественной и антропогенной природы. Поэтому в создании научных и технических основ оценки экологического состояния и риска шельфовых акваторий предлагается использовать данные наблюдений, экосистемные модели и экоскрининговые оценки в качестве моделей усвоения информации. Это открывает возможности экспрессоценок по вероятностным моделям риска. В этом случае результаты расчетов по экосистемным моделям используются как входная информация в моделях риска, и усвоение информации о натурных и дистанционных данных распространяется на все этапы моделирования.

В технократической практике нормирование надежности технических систем ведется из внутренних технических соображений без учета безопасности функционирования экосистем и вариабельности среды. Экоскрининговое нормирование надежности техсистемы ведется относительно биоты. Однако часто считается нормальным, что при неблагоприятных естественных сезонных условиях среды для вероятности аварии технических систем можно допускать более высокие значения, чем при благопри- ятных сезонах с $Q_{m}<Q_{m^{\prime}}$ где $m$ и $m^{\prime}$ соответствуют благоприятным и неблагоприятным естественным сезонным условиям. Это принципиально неверно. Следует надежностные мероприятия по сезонам планировать так, чтобы величины $Q_{m}$ и $Q_{m^{\prime}}$ определялись соотношениями (4).

Грубость оценок риска зависит от объема привлеченной информации об исследуемой акватории. При минимуме такой информации оценки риска становятся тривиальными либо могут возникать ошибки первого и второго рода. Понижение оценки условного риска для групп живых организмов и экосистем по мере понижения неопределенности ситуации (т. е. при проведении необходимых исследований) ведет к уточнению значения допустимой вероятности антропогенных воздействий. Последнее непосредственно связано со снижением надежностных требований к эксплуатируемому оборудованию и расширяет спектр использования различных технологий. Это создает условия для уменьшения затрат на создание, поддержание и функционирование технических систем и технологических процессов при разработке ресурсов арктического шельфа. Использование предложенных методик позволит удовлетворить вынужденным условиям снижения затрат на природоохранные мероприятия при сохранении приоритетности экологических требований.

Работа выполнена в рамках государственного задания, тема № 0149-2019-0005.

\section{Литература}

1. Флинт М. В. Биоресурсы арктических морей России: изменения под воздействием климата и факторов антропогенной природы, экосистемные основы охраны // Научно-технические проблемы освоения Арктики / Рос. акад. наук. - М.: Наука, 2015. - С. 55-71.

2. Комплексные исследования больших морских экосистем России / Отв. ред. Г. Г. Матишов; Мурман. мор. биол. ин-т Кольского науч. центра РАН. - Апатиты: Изд-во КНЦ РАН, 2011. - 516 с.

3. Флинт М. В. 54-й экспедиционный рейс научно-исследовательского судна «Академик Мстислав Келдыш» в Карское море // Океанология. - 2010. Т. 50, № 5. - С. 677-682.

4. Суханова И. Н., Флинт М. В., Мошаров С. А., Сергеева В. М. Структура сообществ фитопланктона и первичная продукция в Обском эстуарии и на прилежащем Карском шельфе // Океанология. 2010. - T. 50, № 5. - C. 785-800.

5. Sukhanova I. N., Flint M. V., Pautova L. A. et al. Phytoplankton of the western Arctic in the spring and summer of 2002: Structure and seasonal changes // DeepSea Res. II. - 2009. - Vol. 56. - P. 1223-1236. DOI: 10.1016/j.dsr2.2008.12.030.

6. Флинт М. В., Семенова Т. Н., Арашкевич Е. Г. и др. Структура зоопланктонных сообществ в области 
эстуарной фронтальной зоны реки Обь // Океанология. - 2010. - Т. 50, № 5. - С. 809-822.

7. Суханова И. Н., Флинт М. В., Дружкова Е. И. и др. Фитопланктон северо-западной части Карского моря // Океанология. - 2015 - Т. 55, № 4. C. 605-619. - DOI: 10.7868/S0030157415040140. 8. Суханова И. Н., Флинт М. В., Сергеева В. М. и др. Структура сообществ фитопланктона Енисейского эстуария и прилежащего Карского шельфа // Океанология. - 2015. - Т. 55, № 6. - С. 935-948. DOI: $10.7868 / S 0030157415060192$.

9. Demidov A. B., Mosharov S. A., Makkaveev P. N. Patterns of the Kara Sea primary production in autumn: Biotic and abiotic forcing of subsurface layer // J. Mar. Sys. - 2014. - Vol. 132. - P. 130-149. DOI: 10.1016/j.jmarsys.2014.01.014.

10. Демидов А. Б., Мошаров С. А., Гагарин В. И., Романова Н. Д. Пространственная изменчивость первичной продукции и хлорофилла «а» в проливе Дрейка в весенний сезон // Океанология. - 2011. - Т. 51, № 2. - C. 293-306.

11. Демидов А. Б., Мошаров С. А. Вертикальное распределение первичной продукции и хлорофилла «а» в Карском море // Океанология. - 2015. T. 55, № 4. - C. 577- 591. - DOI: 10.7868/ S0030157415040024.

12. Демидов А. Б., Мошаров С. А., Артемьев В. А. и Әр. Интегрированные и разрешающие по глубине модели первичной продукции Карского моря // Океанология. - 2016. - Т. 56, № 4. - C. 563-576. - DOI: $10.7868 / \mathrm{S} 003015741604002 \mathrm{X}$.

13. Мошаров С. А., Демидов А. Б., Симакова У. В. Особенности процессов первичного продуцирования в Карском море в конце вегетационного периода // Океанология. - 2016. - Т. 56, № 1. - С. 90100. - DOI: 10.7868/S003015741601010X.

14. Demidov A. B., Kopelevich O. V., Mosharov S. A. et al. Modelling Kara Sea phytoplankton primary production: development and skill assessment of regional algorithms // J. Sea Res. - 2017. - Vol. 125. P. 1-17. - DOI: 10.1016/j.seares.2017.05.004.

15. Демидов А. Б., Шеберстов С. В., Гагарин В. И. и др. Сезонная изменчивость первичной продукции фитопланктона Карского моря по спутниковым данным // Океанология. - 2017. - Т. 57, № 14. - С. 103117. - DOI: 10.7868/S0030157417010026.
16. Ильяш Л. В., Ратькова Т. Н., Радченко И. Г., Житина Л. С. Фитопланктон Белого моря // Система Белого моря. - Т. 2: Водная толща и взаимодействующие с ней атмосфера, криосфера, речной сток и биосфера. - М.: Науч. мир, 2012. - С. 605-639. 17. Беляев В. И. Моделирование морских систем. Киев: Наук. думка, 1987. - 203 с.

18. Беляев В. И. Кондуфорова Н. В. Математическое моделирование экологических систем шельфа. Киев: Наук. думка, 1990. - 240 с.

19. Solovjova N. V. Synthesis of ecosystemic and ecoscreeningmodelling insolving problems ofecological safety//Ecol. Modelling. - 1999. - Vol.124._P. 1-10. 20. Флейшман Б. С. Основы системологии. - М.: Радио и связь, 1982. - 368 с.

21. Флейшман Б. С. Критерии экологической безопасности в условиях неопределенности (экоскрининговый подход) // Экоскрининг: Разработка основ стандартов экологической безопасности. Деп. в ВИНИТИ 19.12.95, № 3401.

22. Соловьева Н. В. Математическое моделирование с использованием данных натурных и дистанционных наблюдений для оценки состояния экологической системы шельфа // Нефтяное хоз-во. - 2005. - № 10. - С. 16-17.

23. Соловьева Н. В., Лобковский Л. И. Исследование чувствительности модели экосистемы шельфа к вариациям ее экологических параметров // Экология пром. пр-ва. - 2008. - № 2. - С. 18-26.

24. Соловьева Н. В. Моделирование кумулятивного влияния внешних факторов на состояние экосистемы шельфа и оценка допустимой вероятности антропогенного воздействия // Эколог. системы и приборы. - 2018. - № 11. - C. 23-35. - DOI: 10.25791/esip.11.2018.253.

25. Соловьева Н. В., Лобковский Л. И. Моделирование эколого-экономического риска при освоении шельфа // ДАН. - 2015. - Т. 464, № 3. - С. 356-360.

26. Соловьева Н. В. Исследования экологической системы шельфа Северного Каспия на основе математического моделирования // Эколог. системы и приборы. - 2016. - № 6. - С. 21-32.

27. Патин С. А. Нефть и экология континентального шельфа: В 2 т. - 2-е изд. - Т. 1: Морской нефтегазовый комплекс: состояние, перспективы, факторы воздействия. - М.: Изд-во ВНИРО, 2017. - 326 с.

\section{Информация об авторах}

Соловьева Наталья Владимировна, доктор физико-математических наук, ведущий научный сотрудник, Институт океанологии им. П. П. Ширшова РАН (117997, Россия, Москва, Нахимовский просп., д. 36), е-таil: soloceanic@yandex.ru.

Лобковский Леопольд Исаевич, доктор физико-математических наук, член-корреспондент РАН, заведующий лабораторией, Институт океанологии им. П. П. Ширшова РАН (117997, Россия, Москва, Нахимовский просп., д. 36), e-mail: Llobkovsky@ocean.ru.

\section{Библиографическое описание данной статьи}

Соловьева Н. В., Лобковский Л. И. Метод оценки экологического риска при освоении ресурсов арктического шельфа // Арктика: экология и экономика. - 2019. — № 1 (33). — С. 50—60. — DOI: 10.25283/2223-4594-2019-1-50-60. 